\title{
An empirical model of submicrocrystalline structure formation and hardening upon severe plastic deformation in iron
}

\author{
M. I. Degtyarev, T. I. Chashchukhina, L. M. Voronova, A. M. Patselov, \\ V. P. Pilyugin and E. F. Talantsev*
}

M.N. Mikheev Institute of Metal Physics, Ural Branch, Russian Academy of Sciences, 18, S. Kovalevskoy St., Ekaterinburg, 620108, Russia

*E-mail: evgeny.talantsev@imp.uran.ru

\begin{abstract}
The evolution of metals micro/nano-structure upon severe plastic deformation (SPD) is still far to be theoretically explained, while experimental datasets are persistently growing for several decades. Major problem associated with understanding of SPD is related to a fact that the latter is a synergistic product of several competing physical effects which alter the material micro/nano-structure. In attempt to find strain boundaries, where predominantly one mechanism determines the micro/nano-structure, in this paper we propose a continuous piecewise model for the analysis of experiments on material hardness vs strain of SPD processed materials. The novelty of this approach lies in its ability to find, as free-fitting parameters, the strain breakpoints which separate different micro/nano-structure modes generated upon SPD process. The model is applied to analyse experimental data for polycrystalline samples of pure iron and two distinctive strain breakpoints are revealed with a good accuracy. This finding is in a good agreement with our earlier results on TEM microscopy studies on pure iron polycrystals after SPD treatment.
\end{abstract}




\section{An empirical model of submicrocrystalline structure formation and hardening upon severe plastic deformation in iron}

\section{Introduction}

The term of severe plastic deformation (SPD) is defined a solid state material treatment in which a very large strain is applied on a bulk sample in order to make an ultra-fine grained material [1]. It should be noted, that from its beginning, SPD is intended to be a part of industrial technology [2] which covers a variety of materials ranging from pure metals [1] to ceramics [3]. Historical review and current development of SPD technology can be found elsewhere [4].

One of main problem associated with the development of quantitative theory of SPD is related to a fact that this materials treatment technique is a synergetic product of several competing physical effects which alter the material micro/nano-structure. In the result, materials structure is not either crystals with long range order, nor amorphous solids with short range order only, but something is in between, as this was first pointed out by Birringer et al [5]. Despite a fact that these authors [5] named nanocrystalline materials as "gas-like solids" (which of course has deep physical meaning), perhaps these days more accurately SPD materials can be named as frozen quantum liquid, because atoms/ions in the SPD solids are in a very strong interaction with each other (and strong particle interaction is not a characteristic of gaseous media).

Remarkably, that the first study of physical properties of SPD materials, which was well ahead even of massive studies of mechanical properties of these materials, was a study of SPD processed quantum materials, i.e. superconducting niobium alloys reported by Fietz and Webb [6]. There is a very interesting issue related to a fact that Fietz and Webb [6] used the term of "severe plastic deformation" in their paper submitted in 1968 which is one year before the report by Langford and Cohen [1]. 
One of milestone result in understanding of SPD is experimental reports on the violation of Hall-Petch relationship in ultrafine grained materials [7-9]. This experimental result is a good accord with our view that attempts to extrapolate physical laws established for microcrystalline materials on SPD materials (with minor modifications) should be failed. However, because the theory of quantum materials is far away to be developed, our current approach is to try to implement some empirical laws which have been found in the field of quantum materials for last 50 years to SPD counterparts.

From a variety of materials on which our attempt can be started, we make a choice for SPD iron. Obviously, iron is considered to be one of model metal [5] in which properties of ultra-fine grained materials can be prominently observed. From other hand, iron is a basic construction material of modern world and, thus, any improvement in iron properties will have a massive impact on global economy.

By considering a property which can be analysed in SPD iron, we take in account that the hardness is one of most conveniently measured property in SPD materials, and thus the establishing of quantitative relation for this property can be interesting for whole R\&D field.

Thus, the purpose of this paper is to reveal a quantitative relation between hardness vs strain in SPD iron. One of our findings is reported herein.

\section{Model description}

In our previous report [10] we showed that some SPD metals exhibit two distinctive microstructures and hardness vs strain dependences, which are attributed to low and high strain. In Fig. 1 we present raw data of hardness vs strain and TEM images of pure iron which is taken from our previous papers $[10,11]$. It can be seen (Fig. 1) that despite distinctive differences in microstructures there is a difficulty to reveal characteristic dependences of hardness vs strain for these two structural states of iron. 


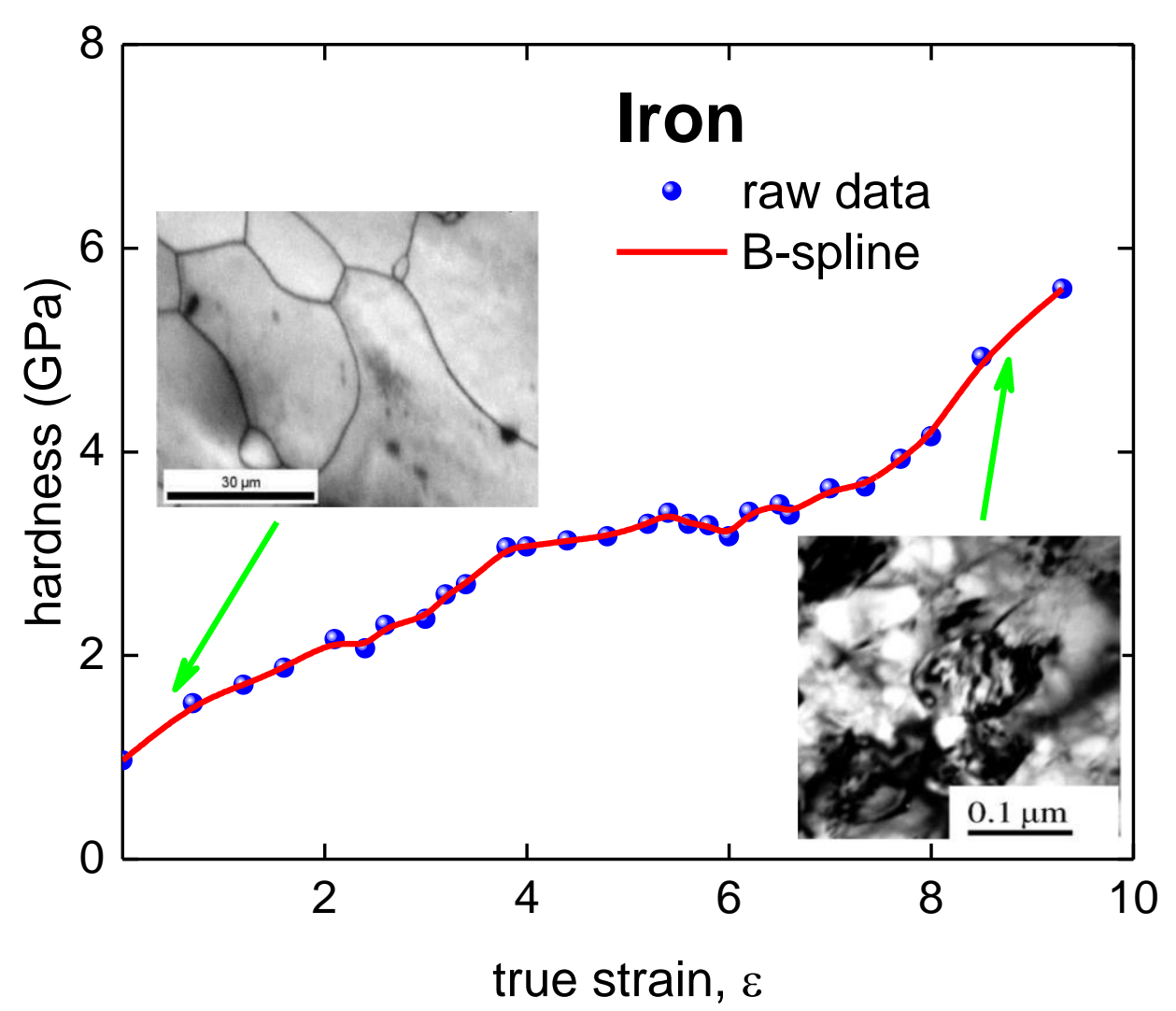

Figure 1. Experimental data of hardness, $H(\varepsilon)$, vs strain and TEM images for structural stage I and structural stage II for pure iron. Raw data and images are taken from Refs. 10,11.

However, as this was first showed by Thompson [8] in pure nickel, and this is also confirmed by us in other metals, that there are two distinctive grains size ranges for each of those a relation between yield strength vs grain size can be established. Thus, our model postulates that:

1. there are two distinctive deformation mechanisms which determine material hardness;

2. there is a strain range where two mechanisms coexist;

3. in this coexisting range of strain, the total hardness is linear additive sum of each mechanism.

These three postulates are expressed in general continuous piecewise fitting function we propose in this paper:

$H(\varepsilon)=\theta\left(\varepsilon_{2}-\varepsilon\right) \cdot H_{1}(\varepsilon)+\theta\left(\varepsilon-\varepsilon_{1}\right) \cdot H_{2}(\varepsilon)+\theta\left(\varepsilon-\varepsilon_{2}\right) \cdot \theta\left(\varepsilon_{1}-\varepsilon\right) \cdot\left(\frac{\left|\varepsilon-\varepsilon_{1}\right|}{\left|\varepsilon_{2}-\varepsilon_{1}\right|} \cdot H_{1}(\varepsilon)+\frac{\left|\varepsilon-\varepsilon_{2}\right|}{\left|\varepsilon_{2}-\varepsilon_{1}\right|} \cdot H_{2}(\varepsilon)\right)$, 
where $H_{1}(\varepsilon)$ and $H_{2}(\varepsilon)$ are hardness functions for structural states I and II respectively (i.e. within strain range of $\left(0, \varepsilon_{1}\right)$ and $\left(\varepsilon_{2}, \infty\right)$ respectively), $\varepsilon_{1}$ is free-fitting upper strain limit for the exhibiting structural state I, and $\varepsilon_{2}$ is free-fitting nucleation breakpoint of structural state II.

For clarity, in Fig. 2 we show the weight function of:

$y=\theta\left(\varepsilon_{2}-\varepsilon\right)+\theta\left(\varepsilon-\varepsilon_{1}\right)+\theta\left(\varepsilon-\varepsilon_{2}\right) \cdot \theta\left(\varepsilon_{1}-\varepsilon\right) \cdot\left(\frac{\left|\varepsilon-\varepsilon_{1}\right|}{\left|\varepsilon_{2}-\varepsilon_{1}\right|}+\frac{\left|\varepsilon-\varepsilon_{2}\right|}{\left|\varepsilon_{2}-\varepsilon_{1}\right|}\right) \equiv 1$

which is splatted in two parts of structural state I and structural state II.

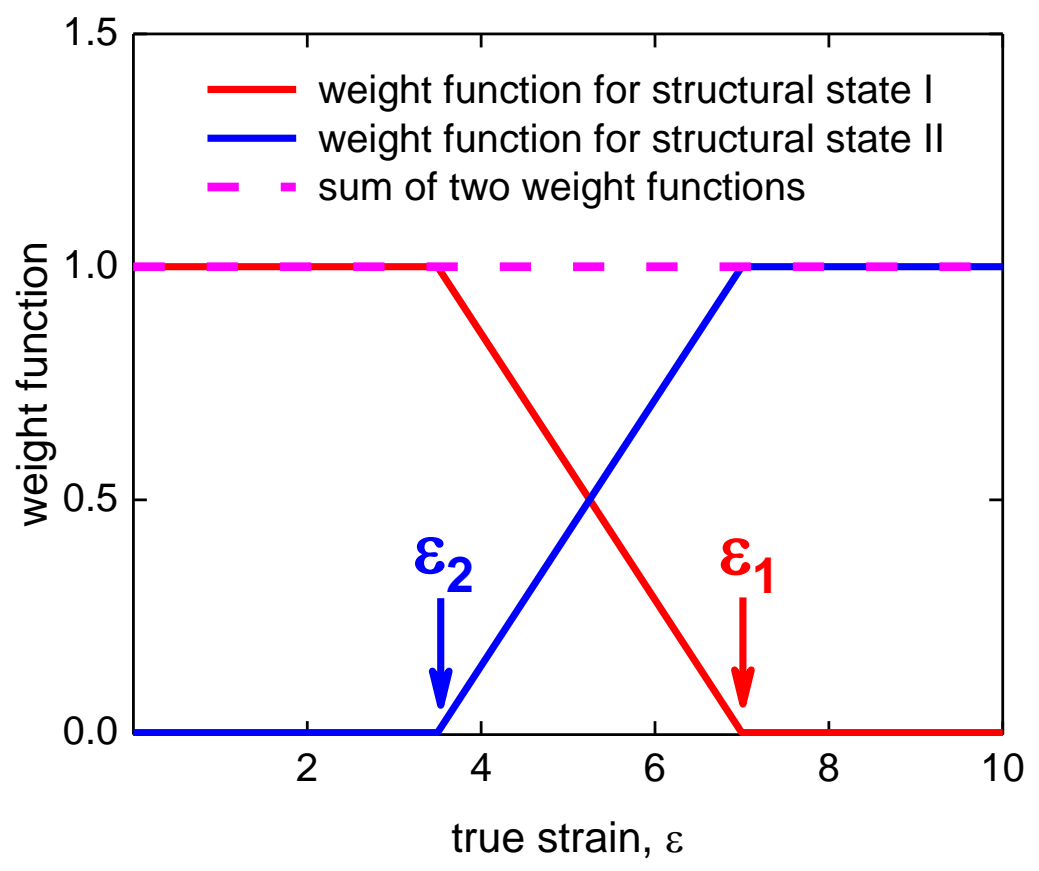

Figure 2. Schematic representation of weight function (Eq. 2) of the model (Eq. 1). $\varepsilon_{1}$ and $\varepsilon_{2}$ are free-fitting breakpoints of the model.

As we already mentioned above, our approach is based on an attempt to implement some empirical laws which have been established for quantum materials (and other complicated physical phenomena, where continuous piecewise function approach is a feasible way to describe the system [12]), for the SPD materials. One of the most widely used approach in quantum materials (and in superconductivity) is to find a scaling law of key property vs one major parameter. In mentioned above paper by Fietz and Webb [6], authors proposed to use 
a scaling in form of power law for the pinning force $F_{\mathrm{p}} \mathrm{vs}$ reduced magnetic field $B / B_{\mathrm{c} 2}$. This approach is in a wide use since then [13-18], as well as decomposition of complicated temperature dependences in superconductivity and normal conductors in a sum of reduced polynomic values $[19,20]$.

Thus, it is very convenient to use analytical form for hardness vs strain function in a form:

$$
\begin{aligned}
& H_{1}(\varepsilon)=H_{1} \cdot\left(1+\left|\frac{\varepsilon}{P}\right|^{\alpha}\right), \text { stage I } \\
& H_{2}(\varepsilon)=H_{2} \cdot\left(1+\left|\frac{\varepsilon}{Q}\right|^{\beta}\right), \text { stage II }
\end{aligned}
$$

where $H_{1}, H_{2}, P, Q, \alpha$ and $\beta$ are free fitting parameters of the model (Eq. 1).

\section{Results and discussions}

Experimental $H(\varepsilon)$ data and fit to Eqs. 1,3 are shown in Fig. 3. Deduced values for $\varepsilon_{1}$ breakpoint (which is the strain of phase I disappearance) and the nucleation breakpoint for the stage II, $\varepsilon_{2}$, are:

$$
\begin{aligned}
& \varepsilon_{1}=5.4 \pm 1.0 \\
& \varepsilon_{2}=3.0 \pm 0.1
\end{aligned}
$$

One of the most interesting result revealed by the fit is that extrapolated $H_{2}(\varepsilon)$ curve (Eq. 3), which is shown in Fig. 3,b (by letter B), which has starting (nucleation) breakpoint at $\varepsilon_{2}=$ $3.0 \pm 0.1$ has absolute value for hardness, $H_{2}(\varepsilon=3.0 \pm 0.1)=4.4 \pm 0.1 \mathrm{GPa}$, which is more or less equal to the ultimate hardness of the material at the maximal strain $\varepsilon_{2} \gtrsim 9 G P a$, before mechanical break. This means that, newly nucleated cellular nanocrystals in SPD iron have defect-free structure which exhibits maximal mechanical properties.

It should be also noted, that deduced power law exponents, $\alpha$ and $\beta$ :

$$
\alpha=0.63 \pm 0.13 \cong \frac{2}{3}
$$




$$
\beta=2.1 \pm 0.3 \cong 2
$$

are in a good agreement with theoretical predicted values for SPD body-centred cubic metals [21].

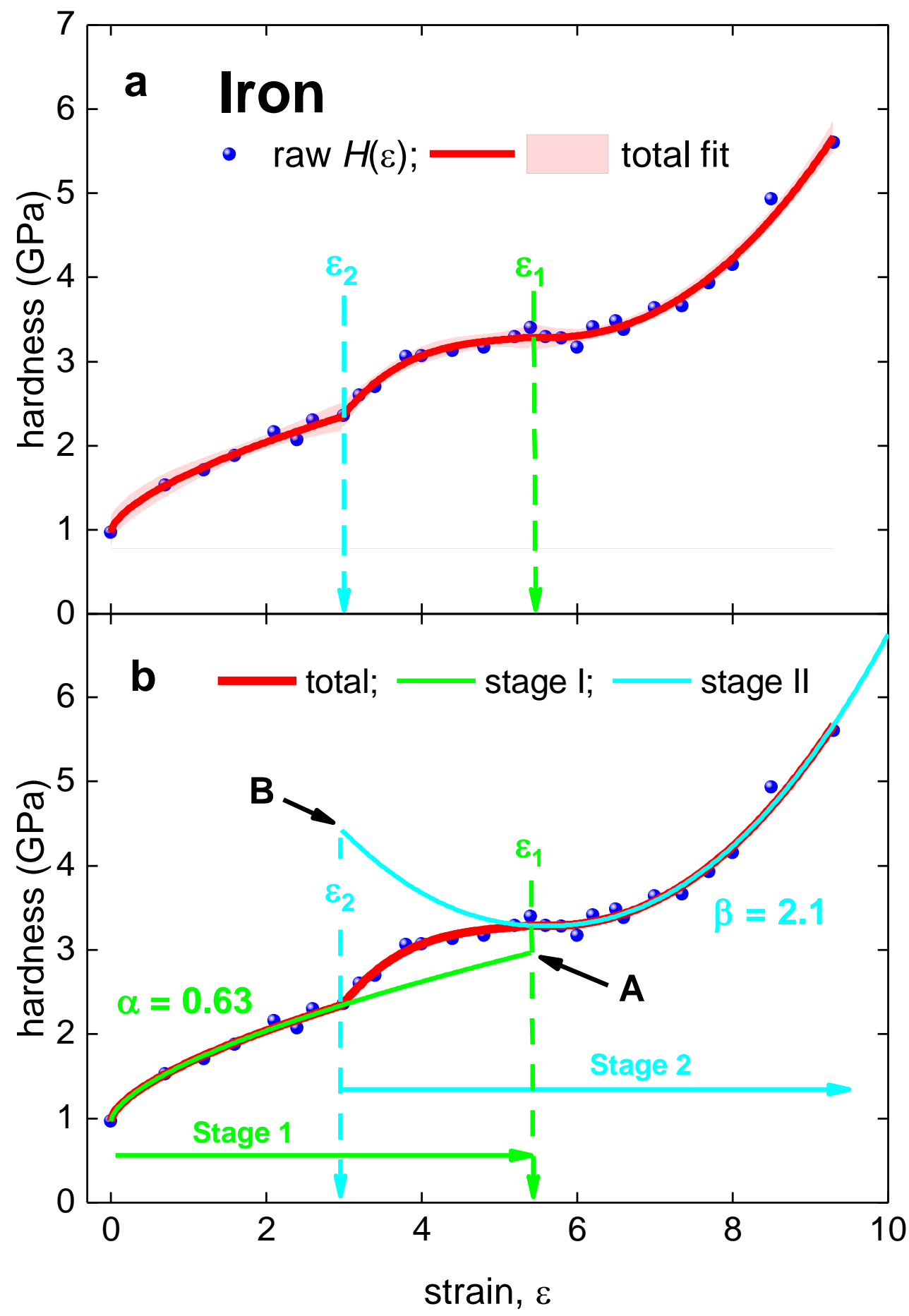

Figure 3. Experimental data of hardness, $H(\varepsilon)$, vs strain and fit to Eqs. 1,3 for pure iron. (a) Total fit, breakpoints $\varepsilon_{1}$ and $\varepsilon_{2}$, and $95 \%$ confidence bars are shown; the fit quality is $R=$ 0.994. (b) Fit where contributions from both stages in overlapped strain range and power law exponent are shown. 


\section{Conclusions}

In this paper we propose a continuous piecewise model for the analysis of experimental hardness vs strain data for SPD processed materials. The novelty of this approach is lied in its ability to deduce, as free-fitting parameters, strain breakpoints which separate different micro/nano-structure modes generated upon SPD process.

We have applied the model to analyse experimental data for polycrystalline samples of pure iron. The analysis reveals distinctive strain boundary of $\varepsilon=3.0 \pm 0.1$ at which cellular nano-crystalline structure starts to form.

\section{Acknowledgement}

Authors thank financial support provided by the state assignment of Minobrnauki of Russia (theme "Pressure" No. AAAA-A18-118020190104-3).

\section{References}

[1] Langford G and Cohen M 1969 Strain hardening of iron by severe plastic deformation Trans. ASM 62 623-638

[2] Gusenkov A P, Zatsarinnyi V V, Shneiderovich R M 1971 Method for low-cycle deformation and failure resistance determination by transverse deformation measurements Industrial Laboratory 37 596-601

[3] Karch J, Birringer R and Gleiter H 1987 Ceramics ductile at low temperature Nature 330 556-558

[4] Valiev R Z, Estrin Y, Horita Z, Langdong T G, Zehetbauer M J and Zhu Y T 2016 Fundamentals of superior properties in bulk nanoSPD materials Mater. Res. Lett. 4 1-21 [5] Birringer R, Gleiter H, Klein H-P, Marquardt P 1984 Nanocrystalline materials an approach to a novel solid structure with gas-like disorder? Physics Letters A 102 365-369 [6] Fietz W A and Webb W W 1969 Hysteresis in superconducting alloys - Temperature and field dependence of dislocation pinning in niobium alloys Physical Review 178 657-667 [7] Anderson E, King D L W and Spreadborough J 1968 The relationship between lower yield stress and grain size in Armco iron. Trans. TMS-AIME 242115

[8] Thompson A W 1975 Yielding in nickel as a function of grain or cell size Acta Metallurgica 23 1337-1342

[9] Chokshi A H, Rosen A, Karch J and Gleiter H 1989 On the validity of the Hall-Petch relationship in nanoscrystalline materials Scripta Metallurgica 23 1679-1684 
[10] Degtyarev M V, Chashchukhina T I, Voronova L M, Patselov A M and Pilyugin V P 2007 Influence of the relaxation processes on the structure formation in pure metals and alloys under high-pressure torsion Acta Materialia 55 6039-6050

[11] Voronova L M, Degtyarev M V, Chashchukhina T I, Shinyavskii D V,Gapontseva T M 2017 Effect of microcrystallites formed by deformation on the growth and orientation of grains during recrystallization of iron Letters on Materials 7 359-362

[12] Riahi M K, Qattan I A, Hassan J and Homouz D 2019 Identifying short- and long-time modes of the mean-square displacement: An improved nonlinear fitting approach AIP Advances 9055112

[13] Hänisch J, et al 2015 High field superconducting properties of $\mathrm{Ba}\left(\mathrm{Fe}_{1-\mathrm{x}} \mathrm{Co}_{\mathrm{x}}\right)_{2} \mathrm{As}_{2}$ thin films Scientific Reports 517363

[14] Poole P P, Farach H A, Creswick R J, Prozorov R 2007 Superconductivity (2-nd Edition, London, UK)

[15] Baumgartner T, Eisterer M, Weber H W, Fluekiger R, Scheuerlein C, Bottura L 2014 Effects of neutron irradiation on pinning force scaling in state-of-the-art $\mathrm{Nb}_{3} \mathrm{Sn}$ wires Supercond. Sci. Technol. 27015005

[16] Li X, et al 2018 Pressure-induced phase transitions and superconductivity in a black phosphorus single crystal PNAS $1159935-9940$

[17] Cai C, et al 2004 Magnetotransport and flux pinning characteristics in $R \mathrm{Ba}_{2} \mathrm{Cu}_{3} \mathrm{O}_{7-\delta}(R=$ $\mathrm{Gd}, \mathrm{Eu}, \mathrm{Nd})$ and $\left(\mathrm{Gd}_{1 / 3} \mathrm{Eu}_{1 / 3} \mathrm{Nd}_{1 / 3}\right) \mathrm{Ba}_{2} \mathrm{Cu}_{3} \mathrm{O}_{7-\delta}$ high- $T_{\mathrm{c}}$ superconducting thin films on $\mathrm{SrTiO}_{3}$ (100) Phys. Rev. B 69104531

[18] Oveshnikov L N, et al 2020 Superconductivity and Shubnikov-de Haas effect in polycrystalline $\mathrm{Cd}_{3} \mathrm{As}_{2}$ thin films Scientific Reports 104601

[19] Kim Y J, et al 2005 Electrical conductivity of chemically modified multiwalled carbon nanotube/epoxy composites Carbon 43 23-30

[20] Zare Y and Rhee K Y 2020 Definition of "b" exponent and development of power-law model for electrical conductivity of polymer carbon nanotubes nanocomposites Results in Physics 16102945

[21] Trefilov V I, Moiseev V F, Pechkovskii E P. In: Deformational strengthening and fracture of metals. Kiev: Naukova Dumka; 1987. p. 242 [in Russian]. 